\title{
Operating with external arguments in the Mandelbrot set antenna
}

\author{
G. Pastor*, M. Romera, G. Álvarez, F. Montoya \\ Instituto de Física Aplicada, Consejo Superior de Investigaciones Científicas, \\ Serrano 144, 28006 Madrid, Spain
}

\begin{abstract}
The external argument theory of Douady and Hubbard allows us to know both the potential and the field-lines in the exterior of the Mandelbrot set. Nonetheless, there are no explicit formulae to operate with external arguments, and the external argument theory is difficult to apply. In this paper we introduce some tools in order to obtain formulae to operate with external arguments in the Mandelbrot set antenna. Thus, we introduce the harmonic tool to calculate both the external arguments of the period doubling cascade hyperbolic components and the external arguments of the last appearance hyperbolic components. Likewise, we introduce composition rules applied to external arguments that, with the aid of the concept of heredity, allows the calculation of all the external arguments that constitutes the family tree of a given external argument. ${ }^{1}$
\end{abstract}

Key words: Mandelbrot set antenna; external arguments; external rays. PACS: 05.45.Ac, 47.20.Ky.

\section{Introduction}

As is known, Douady and Hubbard studied the Mandelbrot set $\mathcal{M}[1,2]$, a bounded set of complex numbers, by computing both the potential created by the set and the external arguments of the points on its boundary $[3,4,5]$. Douady himself divulged the external argument theory as follows [6]. Imagine a capacitor made of an aluminum bar shaped in such a way that its crosssection is $\mathcal{M}$, placed along the axis of a hollow metallic cylinder. Set the bar at potential 0 and the cylinder at a high potential. This creates an electric field

1 This paper was published in Physica D 171 (2002) 52-71

* Telephone: +34-915 618 806; Telefax: +34-914 117 651; Email: gerardo@iec.csic.es 
in the region between the cylinder and the bar. An electric potential function is also established in this region. Assume that the radius of the cylinder is large (with respect to the chosen unit), that its height is large compared to its radius and that the length of the bar is equal to this height. We restrict our attention to the plane perpendicular to the axis of the cylinder, through its middle. In this plane, the electric potential defines equipotential lines enclosing the set $\mathcal{M}$ (which is the cross section of the bar). Following the electric field, one gets field-lines, called the external rays of $\mathcal{M}$. Each external ray starts at a point $x$ on the boundary of $\mathcal{M}$, and reaches a point $y$ of the great circle which is the cross section of the cylinder (practically at infinity). The position of $y$ is identified by an angle, called the external argument of $x$ with respect to the $\mathcal{M}$ set. If there are several accesses to $x$ from outside of $\mathcal{M}$ (for instance, if $\mathcal{M}$ is tree-like and $x$ is a branch point of $\mathcal{M}$ ), then there is one external ray in each access and the point $x$ has several external arguments.

In Fig. 1, the $\mathcal{M}$ set is shown with some of its equipotential lines and external rays. The rational numbers in the figure correspond to the values of the external arguments of the points of $\mathcal{M}$ where the external rays land. The unity for external arguments is the whole turn, not the radian. An external ray starting at a point $x$ on the boundary of $\mathcal{M}$ is identified by the external argument of $x$, and all the points of this external ray have the same external argument.

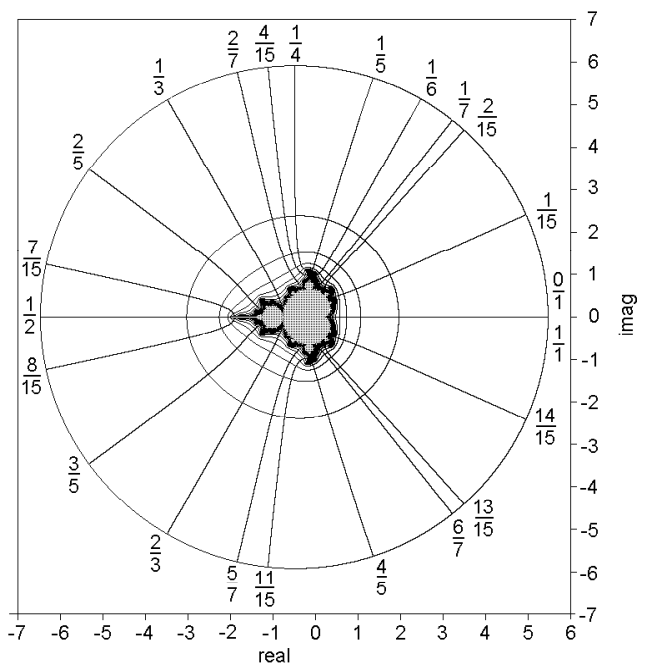

Fig. 1. Equipotential lines, external rays and external arguments in the Mandelbrot set.

As is well known, the Mandelbrot set is defined as the set of points $c$ of the complex plain for which the sequence $c, c^{2}+c,\left(c^{2}+c\right)^{2}+c, \ldots$ does not tend to $\infty$ [7]. This sequence is originated by the iteration of the complex quadratic polynomial $P_{c}(z)=z^{2}+c$. Let us consider the above model of Douady and Hubbard. The potential $U(c)$ of a point $c$ in the interior of the capacitor but outside the Mandelbrot set, is given by the Green's function 


$$
U(c)=\log |c|+\sum_{n=1}^{\infty} \frac{1}{2^{n}} \log \left|1+\frac{c}{P_{c}^{n-1}(c)^{2}}\right|,
$$

where $P_{c}^{0}(c)=c, P_{c}^{1}(c)=c^{2}+c, P_{c}^{2}=\left(c^{2}+c\right)^{2}+c, \ldots$ [8]. The base of the logarithm does not matter. In fact, Eq. (1) ignores the factor of proportionality that depends on the potential of the hollow metal cylinder. We use logarithms to the base 2, according to other authors [9]. Eq. (1) gives potential zero at points of the boundary of the Mandelbrot set. For a large $c$, Eq. (1) reduces to $U(c)=\log |c|$. The locus of points in the complex plane having all of them a same potential is a simple closed curve surrounding the Mandelbrot set. This curve is an equipotential line that consists of values of $c$ for which 0 escapes to $\infty$ at a fixed rate [7].

As is known, the external argument $\theta(c)$ of the external ray that pass through a point $c$, for large $|c|$ is given by the argument of the function $\Phi(c)$ [10]

$$
\Phi(c)=c \prod_{n=1}^{\infty}\left[1+\frac{c}{P_{c}^{n-1}(c)^{2}}\right]^{2^{-n}} .
$$

Taking into account that $\arg (a b)=\arg (a)+\arg (b)$ we have

$$
\theta(c)=\arg (c)+\sum_{n=1}^{\infty} \frac{1}{2^{n}} \arg \left[1+\frac{c}{P_{c}^{n-1}(c)^{2}}\right]
$$

where $\arg (c)$ denotes the principal value of the argument of a complex number, which takes values between $-\pi$ and $\pi$. In this paper we draw external rays using an algorithm from Jung $[11,12]$. The locus of points in the complex plane that have all a same external argument is an external ray. Douady and Hubbard found an algorithm to compute the two external arguments of a point $c$ on the Mandelbrot set antenna [5], as we explain next. We take a point $c^{*}$ next to $c$, over the antenna, obtaining the sequence of arguments $\left\{\arg \left(c^{*}\right), \arg \left(c^{* 2}+c^{*}\right), \arg \left(\left(c^{* 2}+c^{*}\right)^{2}+c^{*}\right), \ldots\right\}$. Next we replace each argument by " 0 " (if it is greater than 0 and less than $\pi$ ) or " 1 " (if it is greater than $\pi$ and less than $2 \pi$ ). In this way we obtain the binary expansion of one of the external arguments of $c$, that is easy to convert at a rational number. The another external argument of $c$ is obtained in the same manner, taking a new point $c^{*}$ next to $c$, under the antenna. Thus, the external arguments of the Misiurewicz point $c=-1.543689012 \ldots$ are $5 / 12$ and $7 / 12$, which we write as the pair $\left(\frac{5}{12}, \frac{7}{12}\right)$.

In this work we will develop formulae to operate with external arguments in the Mandelbrot set antenna. Initially we have obtained Eqs. (4), (6) and (9) 
directly, in an experimental context, from computer calculations. For example we wrote Eq. (4) after finding experimentally the external arguments of the harmonics of many hyperbolic components. The numerators of the external arguments of the harmonics were determined by a system of trial and error, drawing several rays with a computer program by Jung [12] and choosing what corresponded to the harmonics. Later, we have justified these equations. With these formulae we can better understand the "raison d'être", the physical meaning, of a given pair of external arguments corresponding to a point on the Mandelbrot set antenna. For example, as we will see later, the external arguments of the above Misiurewicz point can be obtained, without knowing its parameter value $c$, starting from the well known external arguments of both the root point of period-3 midget $\left(\frac{3}{7}, \frac{4}{7}\right)$ and the root point of period-2 disk $\left(\frac{1}{3}, \frac{2}{3}\right)$. We will obtain the external arguments of this Misiurewicz point as the rightward composition of the external arguments of the only period-3 antenna midget with the external arguments of the period-2 disk an infinite number of times. What we call "midget" is called "mini Mandelbrot set"[7], "tuned copy" [13] or "baby Mandelbrot set" [14] by other authors.

Each one of the Mandelbrot set disks has a root point which connects with a bigger disk or with a cardioid. Each one of the Mandelbrot set cardioids, except for the main cardioid, has a cusp point from which a filament that connects it with the set emerges. Both root points and cusp points are notable points of the boundary of the Mandelbrot set, in the same way as Misiurewicz points which usually are either ramification nodes or tips of the filaments. Other notable points, as the Myrberg-Feigenbaum point, are not studied here.

External arguments are not measured in radians, but as fractions of complete turns. Using this unit, most of the notable points of the Mandelbrot set boundary have rational external arguments. Figure 2 shows the neighborhood of the Mandelbrot set antenna with four examples of notable points and their external rays. The first one on the right is a root point (where the main cardioid and the first disk of the period-doubling cascade join together) which has two rational external arguments with odd denominator $\left(\frac{1}{3}, \frac{2}{3}\right)$. Even though external arguments come from the root point, we also use this pair of external arguments in order to denominate the whole disk that is born at this root point. The second one is the Myrberg-Feigenbaum point, with two irrational external arguments $\theta_{\mathrm{MF}}$ and $1-\theta_{\mathrm{MF}}$ that we shall not study here (as with irrational external arguments it is not clear if the external ray is landing at a well-defined boundary point of the Mandelbrot set). The third one is a Misiurewicz point which as two rational external arguments with even denominator, $\left(\frac{5}{12}, \frac{7}{12}\right)$. The fourth and last one is a cusp point (where the only antenna period-3 cardioid is born) which has two rational external arguments with odd denominator, $\left(\frac{3}{7}, \frac{4}{7}\right)$. Again, even though external arguments come from the cusp point, we also use this pair of external arguments in order to 
denominate the whole cardioid that is born at this cusp point.

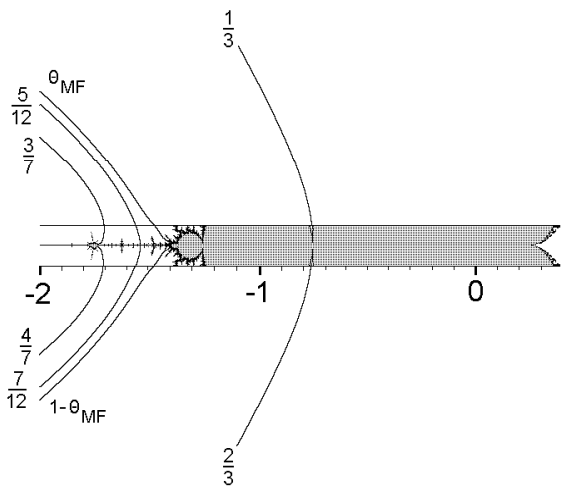

Fig. 2. Mandelbrot set antenna: examples of notable points and their external rays.

As is known, a rational angle $\theta$ with odd denominator is periodic under the doubling function given by $\mathrm{D}(\theta)=2 \theta \bmod 1$. For example, by doubling $1 / 5$ we obtain $1 / 5,2 / 5,4 / 5,3 / 5,1 / 5, \ldots$ which is periodic. Likewise, a rational angle with even denominator is preperiodic under the doubling function. For example, by doubling $1 / 6$ we obtain $1 / 6,2 / 6,4 / 6,2 / 6, \ldots$ which is preperiodic. As is known, in the Mandelbrot set we have periodic and preperiodic points. Root and cusp points are periodic, but Misiurewicz points are preperiodic. Periodic points have periodic external arguments (rational ones with odd denominator), and preperiodic points have preperiodic external arguments (rational ones with even denominator).

The Mandelbrot set is without any doubt the most beautiful example of fractal structure generated by nonlinear dynamical systems. Nonlinear dynamical systems have been broadly treated in all disciplines, especially in physics because of their wide range of applications in a large number of nonlinear problems. Thus, the first physically motivated study of chaotic dynamics was presented in 1963 by Lorenz [15], and in 1971 Ruelle and Takens suggested that turbulent flow might be an example of dynamical chaos [16]. In following years dynamical systems were applied to almost any branch of the physics from pattern formation in natural systems [17] to fluid mechanics [18]. Nowadays, the interest on this topic continues as we can see for example in [19]. Therefore, we reckon that studying nonlinear dynamical systems in the widest possible manner should positively contribute to enrich physical research.

We have studied one-dimensional (1D) quadratic maps by means of the hyperbolic components [7], Misiurewicz points [20] and other notable points of the Mandelbrot set which intersect with the real axis [21, 22, 23, 24, 25, 26, 27]. Figure 2 shows the neighborhood of this Mandelbrot set subset, that we call "antenna". This subset has an enormous interest since it is the portrait of the $1 \mathrm{D}$ quadratic map $x_{n+1}=x_{n}^{2}+c$, and can be used to study this map. In previous papers we have studied hyperbolic components and Misiurewicz points 
of the Mandelbrot set antenna by using their symbolic sequences [28, 29] (or keading sequences according to other authors [30]). In this paper we shall study the Mandelbrot set antenna by using the external arguments of Douady and Hubbard instead of the symbolic sequences we used to use.

In a previous paper, we based on the Metropolis, Stein and Stein (MSS) harmonics [31] to introduce other harmonics what we called there Fourier harmonics [21] though later we have called them F-harmonics or simply harmonics. By using this harmonic tool we could calculate the symbolic sequences of all the structural components (and the Misiurewicz points that separates them) of the 1D quadratic maps; i. e., we could determine the structure of such maps [23].

In the first part of this paper we shall apply the harmonic tool for the case of external arguments, in such a manner that we can calculate the external arguments of the structural hyperbolic components and the Misiurewicz points that separate them. I. e., we want to calculate the structure of the Mandelbrot set antenna, but now given in external arguments.

Likewise, in other previous paper [24] we introduced the concept of heredity in $1 \mathrm{D}$ quadratic maps. The heredity concept is not consequence of a theorem but a conclusion reached on the basis of a lot of computer experiments. As we showed there, given a hyperbolic component we can calculate the ancestral path that joins all its ancestors, and from these ancestors we can calculate all the descendants of the hyperbolic component, i.e. the family tree. In order to do so, we need to use the composition rules of the symbolic sequences that were introduced by us [23]. In this paper we also introduce new composition rules, now applied to external arguments. With these composition rules for external arguments we will be able, by using the heredity concept, of calculating all the external arguments that constitutes the family tree of a given external argument.

To begin with, let us see how to operate with external arguments of the Mandelbrot set antenna in a similar way as we did with symbolic sequences in our previous papers. In such a way we can calculate the external arguments of the hyperbolic components and Misiurewicz points of the antenna.

\section{MSS-harmonics of a hyperbolic component}

Let us consider a period- $p$ hyperbolic component of the Mandelbrot set antenna. If it is a disk, it is connected to the antenna through its tangent point (root point); and, if it is a cardioid, through its cusp. When we start from a period- $p$ hyperbolic component, and we progress through its period doubling 
cascade, as is well known we find disks whose periods are $2^{1} p, 2^{2} p, 2^{3} p, \ldots$. In 1973 MSS introduced the concept of harmonic in 1D unimodal maps [31]. By extension, we call "MSS-harmonics" of a hyperbolic component of the Mandelbrot set antenna to the set constituted by itself and the disks of its period doubling cascade. The MSS-harmonics were an important achievement because, starting from the symbolic sequence (or pattern) of a period- $p$ hyperbolic component we can calculate the pattern of all the disks of its period doubling cascade. Thus, the $i^{t h}$ MSS-harmonic is the $i^{t h}$ disk of its period doubling cascade, which has a period $2^{i} p$, and therefore a pattern that is $2^{i}$ times long the original. As we said before, we have applied these concepts to the case of external arguments.

Let $\left(\frac{a_{1}}{2^{p}-1}, \frac{a_{2}}{2^{p}-1}\right)$ be the normalized external arguments of a period- $p$ hyperbolic component. As is known, it is often useful to consider the sequence of binary digits. $\bar{a}$ of a periodic external argument $\frac{a}{2^{p}-1}$. Here the number a is interpreted as a sequence of $p$ binary digits. For example in $6 / 15$, with $p=4$, it is understood that $6=0110$ and we write $6 / 15=. \overline{0110}$. Let $\left(. \overline{a_{1}}, . \overline{a_{2}}\right)$ be the binary expansions of the external arguments of a hyperbolic component of the Mandelbrot set antenna. By taking into account that the binary expansions of the period-2 disk are $(. \overline{01}, . \overline{10})$, it is easy to obtain the binary expansions of the external arguments of a MSS-harmonic of $\left(. \overline{a_{1}}, . \overline{a_{2}}\right)$ from the tuning algorithm by Douady and Hubbard [5, 32]. We have

$$
\begin{gathered}
H_{M S S}^{(1)}\left(\overline{a_{1}}, . \overline{a_{2}}\right)=\left(. \overline{a_{1} a_{2}}, \overline{a_{2} a_{1}}\right) \\
H_{M S S}^{(2)}\left(. \overline{a_{1}}, \overline{a_{2}}\right)=\left(. \overline{a_{1} a_{2} a_{2} a_{1}}, \overline{a_{2} a_{1} a_{1} a_{2}}\right), \\
H_{M S S}^{(3)}\left(. \overline{a_{1}}, . \overline{a_{2}}\right)=\left(. \overline{a_{1} a_{2} a_{2} a_{1} a_{2} a_{1} a_{1} a_{2}}, \overline{a_{2} a_{1} a_{1} a_{2} a_{1} a_{2} a_{2} a_{1}}\right),
\end{gathered}
$$

and the pair of normalized external arguments of the order $i$ MSS-harmonic of $\left(\frac{a_{1}}{2^{p}-1}, \frac{a_{2}}{2^{p}-1}\right)$ are given by

$$
\begin{gathered}
H_{M S S}^{(i)}\left(\frac{a_{1}}{2^{p}-1}, \frac{a_{2}}{2^{p}-1}\right)= \\
\left(\frac{a_{1(i-1)} 2^{2^{i-1} p}+a_{2(i-1)}}{2^{2^{i} p}-1}, \frac{a_{2(i-1)} 2^{2^{i-1} p}+a_{1(i-1)}}{2^{2^{i} p}-1}\right), i \geq 1,
\end{gathered}
$$


where $a_{1(0)}=a_{1}$ and $a_{2(0)}=a_{2}$. Equations (3) and (4) also make sense in the complex case, outside the Mandelbrot set antenna.

\section{Example}

The external arguments of the Mandelbrot set main cardioid are $\left(\frac{0}{1}, \frac{1}{1}\right)$. The first four MSS-harmonics of $\left(\frac{0}{1}, \frac{1}{1}\right)$ are the external arguments of the first four disks of the period doubling cascade of such a main cardioid:

$H_{M S S}^{(1)}\left(\frac{0}{1}, \frac{1}{1}\right)=\left(\frac{1}{3}, \frac{2}{3}\right)$,

$H_{M S S}^{(2)}\left(\frac{0}{1}, \frac{1}{1}\right)=\left(\frac{6}{15}, \frac{9}{15}\right)=\left(\frac{2}{5}, \frac{3}{5}\right)$,

$H_{M S S}^{(3)}\left(\frac{0}{1}, \frac{1}{1}\right)=\left(\frac{105}{255}, \frac{150}{255}\right)=\left(\frac{7}{17}, \frac{10}{17}\right)$ and

$H_{M S S}^{(4)}\left(\frac{0}{1}, \frac{1}{1}\right)=\left(\frac{27030}{65535}, \frac{38505}{65535}\right)=\left(\frac{106}{257}, \frac{151}{257}\right)$.

In Fig. 3(a) we have separately depicted each one of the external arguments of the first four disks of the period doubling cascade of the main cardioid. $H_{M S S}^{(\infty)}\left(\frac{0}{1}, \frac{1}{1}\right)$ are the external arguments of the Myrberg-Feigenbaum point $[3,33,34]$. This notable point has not rational external arguments. As is known, the series $1 / 3=0 . \overline{01}, 2 / 5=0 . \overline{0110}, 7 / 17=0 . \overline{01101001}$, $106 / 257=0 . \overline{0110100110010110}, \ldots$ tends to the Thue-Morse constant $\theta_{M F}=$ $0.4124 \ldots$, which is the smaller of the external arguments of the MyrbergFeigenbaum point. Obviously, the series $2 / 3,3 / 5,10 / 17,151 / 257 \ldots$ tends to $1-\theta_{M F}$.

\section{Example}

The external arguments of the only period-3 cardioid of the antenna, located at $c=-1.754877666 \ldots$, are $\left(\frac{3}{7}, \frac{4}{7}\right)$. The first four MSS-harmonics of his cardioid are the first four disks of the period doubling cascade of such a period-3 cardioid:

$H_{M S S}^{(1)}\left(\frac{3}{7}, \frac{4}{7}\right)=\left(\frac{28}{63}, \frac{35}{63}\right)=\left(\frac{4}{9}, \frac{5}{9}\right)$,

$H_{M S S}^{(2)}\left(\frac{3}{7}, \frac{4}{7}\right)=\left(\frac{1827}{4095}, \frac{2268}{4095}\right)=\left(\frac{29}{65}, \frac{36}{65}\right)$,

$H_{M S S}^{(3)}\left(\frac{3}{7}, \frac{4}{7}\right)=\left(\frac{7485660}{16777215}, \frac{9291555}{16777215}\right)=\left(\frac{1828}{4097}, \frac{2269}{4097}\right)$ and

$H_{M S S}^{(4)}\left(\frac{3}{7}, \frac{4}{7}\right)=\left(\frac{125588544014115}{281474976710656}, \frac{155886432696540}{281474976710656}\right)=\left(\frac{7485661}{16777217}, \frac{9291556}{16777217}\right)$.

In Fig. 3(b) we have separately depicted each one of the two external arguments of the first three disks of the period doubling cascade of the period- 


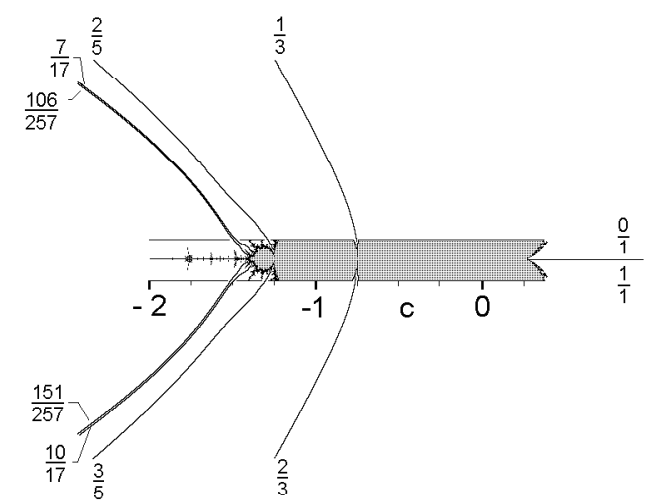

(a)

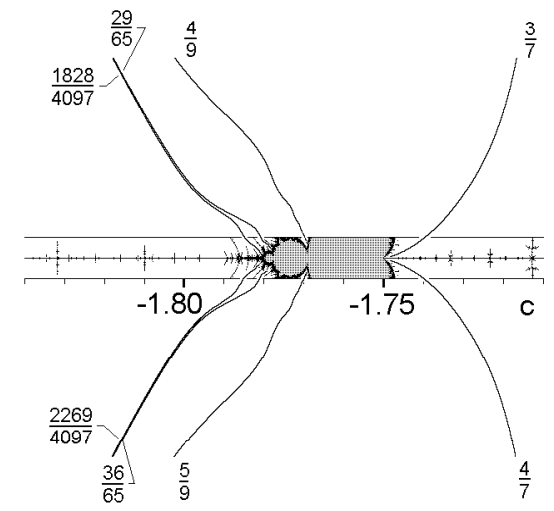

(b)

Fig. 3. Mandelbrot set antenna. (a) MSS-harmonics of the external arguments of the main cardioid. (b) MSS-harmonics of the period-3 cardioid.

3 cardioid. Likewise, $H_{M S S}^{(\infty)}\left(\frac{3}{7}, \frac{4}{7}\right)$ is the limit of the former external arguments, with irrational external arguments that corresponds to the equivalent Myrberg-Feigenbaum point.

As we have seen in the two former examples, a pair of external arguments writen in the normalized form can be simplified by dividing the numerator and denominator of each one of them by $2^{2^{i-1} p}-1$, the irreducible form. Let us suppose that $H_{M S S}^{(i)}\left(\frac{a_{1}}{b}, \frac{a_{2}}{b}\right)=\left(\frac{a_{1(i)}}{b_{(i)}}, \frac{a_{2(i)}}{b_{(i)}}\right)=\left(\frac{\alpha_{1(i)}}{\beta_{(i)}}, \frac{\alpha_{2(i)}}{\beta_{(i)}}\right)$, where $\left(\frac{a_{1(i)}}{b_{(i)}}, \frac{a_{2(i)}}{b_{(i)}}\right)$ is the normalized form and $\left(\frac{\alpha_{1(i)}}{\beta_{(i)}}, \frac{\alpha_{2(i)}}{\beta_{(i)}}\right)$ is the irreducible form. We have the following property: $\left(\frac{\alpha_{1(i+1)}}{\beta_{(i+1)}}, \frac{\alpha_{2(i+1)}}{\beta_{(i+1)}}\right)=\left(\frac{a_{1(i)}+1}{b_{(i)}+2}, \frac{a_{2(i)}+1}{b_{(i)}+2}\right)$. i.e., by knowing the normalized form of $H_{M S S}^{(i)}$ we can directly give the irreducible form of $H_{M S S}^{(i+1)}$ and vice versa, as can clearly be seen in the two former examples. 


\section{Fourier harmonics of a hyperbolic component}

As we have already pointed out before in [26], we have introduced the Fourier harmonics, or F-harmonics, applied to the pattern of a hyperbolic component of a 1D quadratic map. These F-harmonics have a different meaning that the MSS-harmonics. Indeed, as we saw before, the order $i$ MSS-harmonic corresponds to the $i^{t h}$ disk of the period doubling cascade which has a period $2^{i} p$, and therefore a pattern with a length $2^{i}$ times the original. However, the Fourier harmonics, except for the first harmonic, are all of them last appearance cardioids in the corresponding chaotic band [23], and the order $i$ F-harmonic has a period $(i+1) p$, and therefore has a pattern with a length $i+1$ times the original. As we did in the case of the MSS-harmonics, we shall introduce now the F-harmonics of the external arguments of an antenna hyperbolic component. Again we shall see that these harmonics, except for the first one, correspond to the external arguments of the last appearance cardioids of the corresponding chaotic bands.

Let $\left(\frac{a_{1}}{2^{p}-1}, \frac{a_{2}}{2^{p}-1}\right)$ be the pair of the normalized external arguments of a period$p$ hyperbolic component and let $\left(. \overline{a_{1}}, \overline{a_{2}}\right)$ be the corresponding binary extensions. Taking into account that the binary expansions of the last appearance hyperbolic components of periods $2,3,4,5 \ldots$ in the Mandelbrot set antenna are $(. \overline{01}, . \overline{10}),(. \overline{011}, . \overline{100}),(. \overline{0111}, . \overline{1000}),(. \overline{01111}, . \overline{10000}) \ldots$ it is easy to obtain the binary expansions of the external arguments of a F-harmonic of $\left(. \overline{a_{1}}, . \overline{a_{2}}\right)$ from the tuning algorithm by Douady and Hubbard. We have

$$
\begin{gathered}
H_{F}^{(1)}\left(. \overline{a_{1}}, \overline{a_{2}}\right)=\left(. \overline{a_{1} a_{2}}, \overline{a_{2} a_{1}}\right), \\
H_{F}^{(2)}\left(\overline{a_{1}}, \overline{a_{2}}\right)=\left(\overline{a_{1} a_{2} a_{2}}, \overline{a_{2} a_{1} a_{1}}\right), \\
\cdots, \\
H_{F}^{(i)}\left(. \overline{a_{1}}, . \overline{a_{2}}\right)=(. \overline{a_{1}} \underbrace{a_{2} a_{2} \ldots a_{2}}_{i}, \overline{a_{2}} \underbrace{a_{1} a_{1} \ldots a_{1}}_{i}),
\end{gathered}
$$

and the pair of normalized external arguments of the order $i$ F-harmonic are given by 


$$
H_{F}^{(i)}\left(\frac{a_{1}}{2^{p}-1}, \frac{a_{2}}{2^{p}-1}\right)=\left(\frac{a_{1} 2^{i p}+a_{2} \sum_{j=0}^{j=i-1} 2^{j p}}{2^{(i+1) p}-1}, \frac{a_{2} 2^{i p}+a_{1} \sum_{j=0}^{j=i-1} 2^{j p}}{2^{(i+1) p}-1}\right)
$$

Note that the external arguments of both the first F-harmonic and the first MSS-harmonic of a hyperbolic component are the same. Equations (5) and (6) also make sense in the complex case, outside the Mandelbrot set antenna.

\section{Example}

The external arguments of the Mandelbrot set main cardioid are $\left(\frac{0}{1}, \frac{1}{1}\right)$. The four first F-harmonics of $\left(\frac{0}{1}, \frac{1}{1}\right)$ are external arguments of last appearance cardioids, except for the first one which is the first disk of its period doubling cascade. These values are:

$H_{F}^{(1)}\left(\frac{0}{1}, \frac{1}{1}\right)=\left(\frac{1}{3}, \frac{2}{3}\right)$,

$H_{F}^{(2)}\left(\frac{0}{1}, \frac{1}{1}\right)=\left(\frac{3}{7}, \frac{4}{7}\right)$,

$H_{F}^{(3)}\left(\frac{0}{1}, \frac{1}{1}\right)=\left(\frac{7}{15}, \frac{8}{15}\right)$,

$H_{F}^{(4)}\left(\frac{0}{1}, \frac{1}{1}\right)=\left(\frac{15}{31}, \frac{16}{31}\right)$,

$\cdots$.

As we can see in Fig. 4(a), the first F-harmonic corresponds to the first disk of the period doubling cascade while the following three F-harmonics correspond to the period 3, 4 and 5 last appearance cardioids.

Let $\left(\frac{a_{1}}{2^{p}-1}, \frac{a_{2}}{2^{p}-1}\right), a_{2}>a_{1}$, be the normalized external arguments of a period- $p$ hyperbolic componente. By definition, these external arguments have the normalized increment $\frac{a_{2}-a_{1}}{2^{p}-1}$. Each one of the main cardioid F-harmonics is, among all the hyperbolic components with the same period, that which has the smaller normalized increment of the argument. Thus, on the Mandelbrot set antenna there are three period-5 hyperbolic components whose normalized external arguments are $\left(\frac{13}{31}, \frac{18}{31}\right),\left(\frac{14}{31}, \frac{17}{31}\right)$ and $\left(\frac{15}{31}, \frac{16}{31}\right)$; however, only the last one, with the smaller normalized increment of the argument, is a F-harmonic. This pair of external arguments correspond indeed to the fourth main cardioid F-harmonic.

\section{Example}




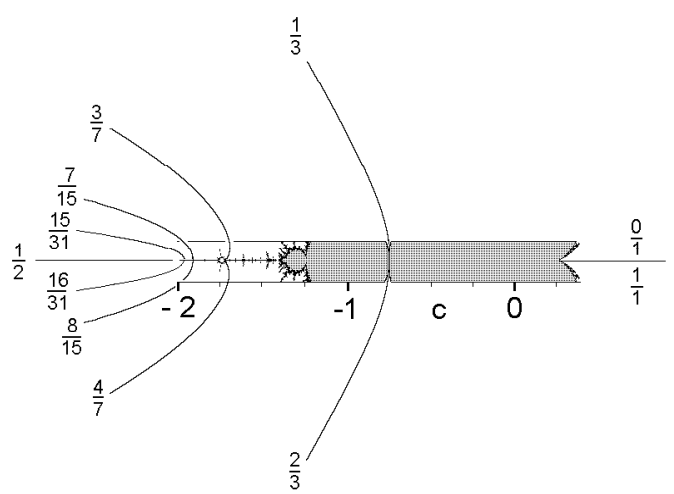

(a)

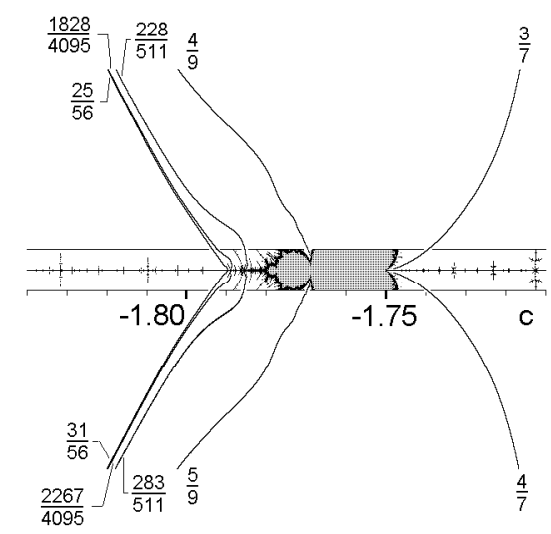

(b)

Fig. 4. Mandelbrot set antenna. (a) F-harmonics of the external arguments of the main cardioid. (b) F-harmonics of the period-3 cardioid.

The four first F-harmonics of the only period-3 antenna cardioid are the first disk of the period doubling cascade (with period 6) and the three last appearance cardioids (with periods 9, 12 and 15) of such a period-3 cardioid:

$H_{F}^{(1)}\left(\frac{3}{7}, \frac{4}{7}\right)=\left(\frac{28}{63}, \frac{35}{63}\right)=\left(\frac{4}{9}, \frac{5}{9}\right)$

$H_{F}^{(2)}\left(\frac{3}{7}, \frac{4}{7}\right)=\left(\frac{228}{511}, \frac{283}{511}\right)$,

$H_{F}^{(3)}\left(\frac{3}{7}, \frac{4}{7}\right)=\left(\frac{1828}{4095}, \frac{2267}{4095}\right)$,

$H_{F}^{(4)}\left(\frac{3}{7}, \frac{4}{7}\right)=\left(\frac{14628}{32767}, \frac{18139}{32767}\right)$,

As we can see In Fig. 4(b), the first F-harmonic corresponds to the first disk of the period doubling cascade while the following two F-harmonics correspond to period-9 and period-12 last appearance cardioids. 


\section{Limit of the Fourier harmonics}

When we studied F-harmonics by means of the symbolic sequences [26], we saw that the order $\infty$ F-harmonic of a cardioid is the tip of such a cardioid, while the order $\infty$ F-harmonic of a disk of the period doubling cascade is a merging point. We shall see next that for the case of external arguments the same happens. The external argument of the order $\infty$ F-harmonic of a hyperbolic component whose external arguments are $\left(\frac{a_{1}}{2^{p}-1}, \frac{a_{2}}{2^{p}-1}\right), a_{2}>a_{1}$, can be calculated as:

$$
H_{F}^{(\infty)}\left(\frac{a_{1}}{2^{p}-1}, \frac{a_{2}}{2^{p}-1}\right)=\left(\frac{a_{1}\left(2^{p}-1\right)+a_{2}}{2^{p}\left(2^{p}-1\right)}, \frac{a_{1}+a_{2}\left(2^{p}-1\right)}{2^{p}\left(2^{p}-1\right)}\right)
$$

Equation (7) has been obtained starting from Eq. (6) when $i$ tends to the infinity. Likewise, if we start from Eq. (5) we obtain $H_{F}^{(\infty)}\left(. \overline{a_{1}}, . \overline{a_{2}}\right)=\left(. a_{1} \overline{a_{2}}, . a_{2} \overline{a_{1}}\right)$.

\section{Example}

Let us calculate the order $\infty$ F-harmonic of the main cardioid whose external arguments are $\left(\frac{0}{1}, \frac{1}{1}\right)$. By applying Eq. (7) we obtain: $H_{F}^{(\infty)}\left(\frac{0}{1}, \frac{1}{1}\right)=\left(\frac{1}{2}, \frac{1}{2}\right)$, that indeed is the only value (double) of the tip located at $c=-2$, as we can see in Fig. 4(a).

\section{Example}

Let us calculate the order $\infty$ F-harmonic of the only period-3 cardioid of the antenna. By applying Eq. (7) we obtain: $H_{F}^{(\infty)}\left(\frac{3}{7}, \frac{4}{7}\right)=\left(\frac{25}{56}, \frac{31}{56}\right)$, that indeed are the external arguments of the tip of the cardioid $\left(\frac{3}{7}, \frac{4}{7}\right)$, located at $c=-1.790327491 \ldots$, as we can see in Fig. 4(b). In these two examples we have seen that the order $\infty$ F-harmonic of a cardioid is the tip of such a cardioid. In the following section we shall see several examples to show that the order $\infty$ F-harmonic of a disk of the period doubling cascade is a merging point.

\section{External arguments of the harmonic structure of the antenna}

In order to calculate symbolic sequences in 1D quadratic maps F-harmonics are a very powerful tool since starting from the main cardioid, of simbolic sequence $\mathrm{C}$, we can generate the successive chaotic bands and calculate the pattern of any of their structural components (last appearance hyperbolic components) [23]. 
As we can see in [23], the first F-harmonic of the main cardioid is the period-2 disk of the period-doubling cascade. All the other F-harmonics of the main cardioid are cardioids placed in the period $-2^{0}$ chaotic band $\mathbf{B}_{0}$ and are the last appearance cardioids of this chaotic band. If we consider the period- $2^{0}$ main cardioid as a "gene" $\mathbf{G}_{0}$, then the F-harmonics of $\mathbf{G}_{0}$ generate the structure of the chaotic band $\mathbf{B}_{0}$. The limit of the band $\mathbf{B}_{0}$ is the Misiurewicz point $m_{0}$, that is the antenna tip located at $c=-2$.

We are in the same scenario in the case of external arguments. Indeed, if we start from the external arguments $\left(\frac{0}{1}, \frac{1}{1}\right)$ of the main cardioid $\mathbf{G}_{0}$ and we calculate its successive F-harmonics according to Eq. (6), we have $H_{F}^{(1)}\left(\mathbf{G}_{0}\right)=$ $\left(\frac{1}{3}, \frac{2}{3}\right), H_{F}^{(2)}\left(\mathbf{G}_{0}\right)=\left(\frac{3}{7}, \frac{4}{7}\right), H_{F}^{(3)}\left(\mathbf{G}_{0}\right)=\left(\frac{7}{15}, \frac{8}{15}\right), \ldots$. As we show in Fig. 5, $H_{F}^{(1)}\left(\mathbf{G}_{0}\right)$ corresponds to the first disk of the period-doubling cascade. All the other /F-harmonics $H_{F}^{(2)}\left(\mathbf{G}_{0}\right), H_{F}^{(3)}\left(\mathbf{G}_{0}\right), \ldots$, are cardioids placed in the chaotic band $\mathbf{B}_{0}$ and are the last appearance cardioids of this band. The limit of the band $\mathbf{B}_{0}$, calculated according to Eq. (7), is $H_{F}^{(\infty)}\left(\mathbf{G}_{0}\right)=H_{F}^{(\infty)}\left(\frac{0}{1}, \frac{1}{1}\right)=$ $\left(\frac{1}{2}, \frac{1}{2}\right)$ that indeed corresponds to the Misiurewicz point $m_{0}$ that is the antenna tip located at $c=-2$. At the tip both external arguments coincide in only one, but double, in such a way that $\frac{1}{2}+\frac{1}{2}=1$ (see Fig.6).

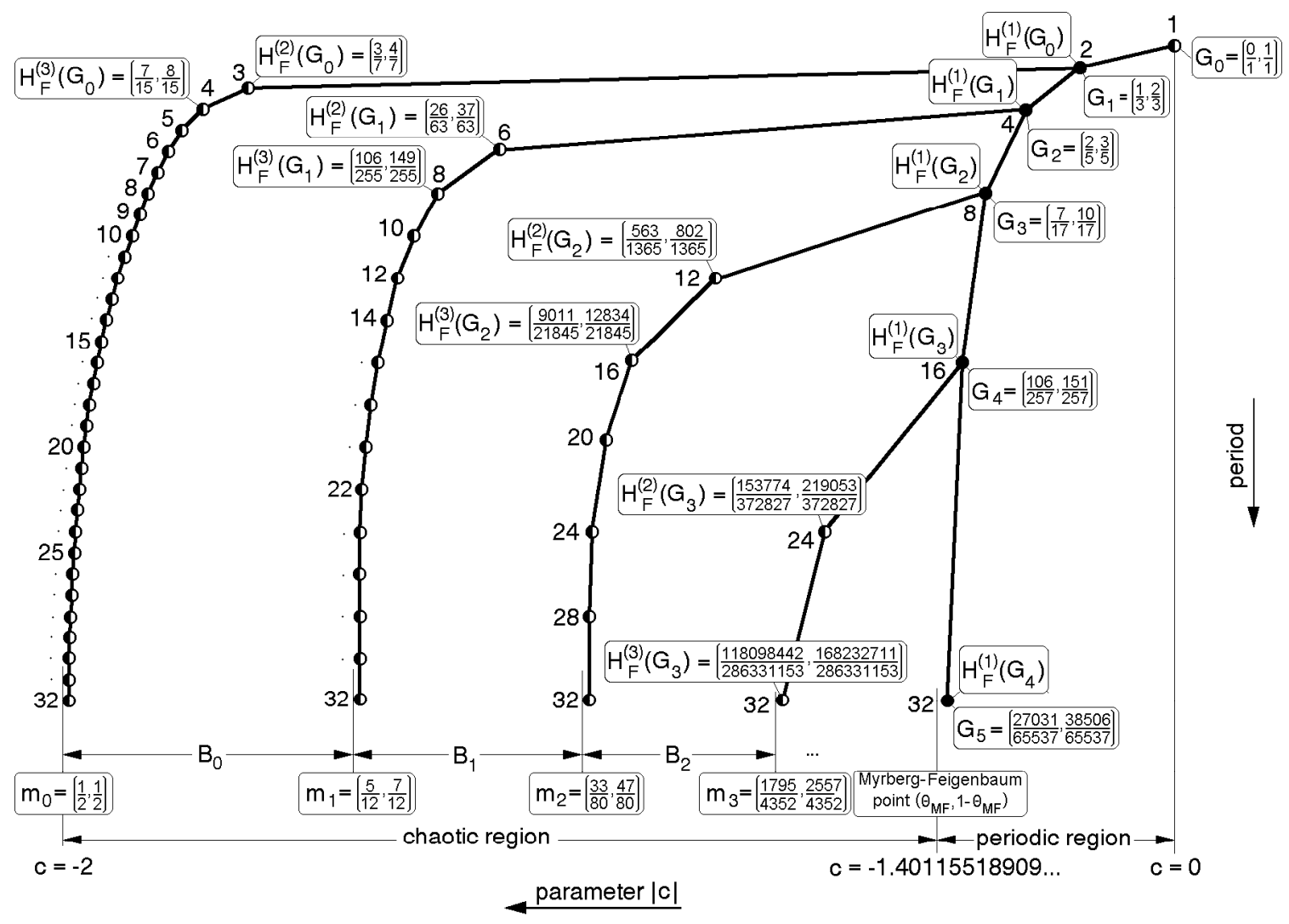

Fig. 5. External arguments of the harmonic structure of the Mandelbrot set antenna. 


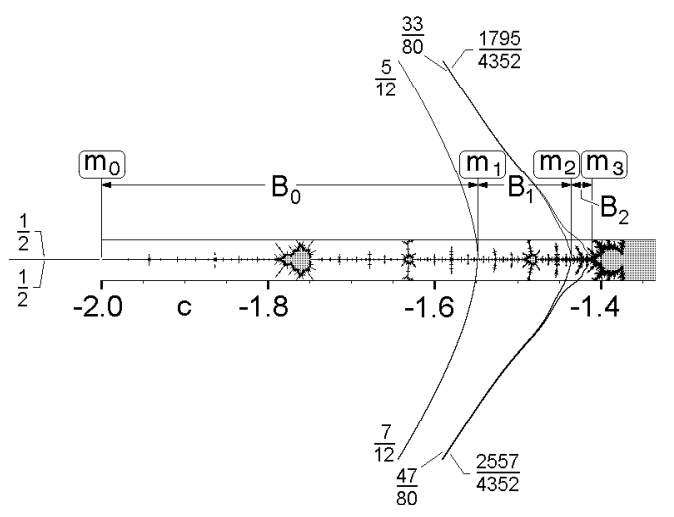

Fig. 6. Mandelbrot set antenna. Chaotic bands $\mathbf{B}_{0}, \mathbf{B}_{1}, \mathbf{B}_{2}$ and external arguments of the Misiurewicz points $m_{0}, m_{1}, m_{2}$ and $m_{3}$.

The first F-harmonic of $\mathbf{G}_{0}$ is not a cardioid but a period- $2^{1}$ disk placed in the Feigenbaum region, that we shall call "bridge pattern". Let us remember what happens when this disk is used as a new gene in [23]. We can consider this "bridge pattern" as a new gene $\mathbf{G}_{1}$. Then, we have that the F-harmonics of the gene $\mathbf{G}_{1}$ generate the structure of the period- $2^{1}$ chaotic band $\mathbf{B}_{1}$ (and a new bridge pattern that will be the second disk of the period-doubling cascade, the gene $\mathbf{G}_{2}$ ). The limit of the band $\mathbf{B}_{1}$ is the Misiurewicz point $m_{1}$, located at $c=-1.543689012 \ldots$, that separates the chaotic bands $\mathbf{B}_{0}$ and $\mathbf{B}_{1}$.

For external arguments, if we start from the external argument of the gene $\mathbf{G}_{1}=\left(\frac{1}{3}, \frac{2}{3}\right)$ and we calculate the successive F-harmonics according to Eq. (6), we have $H_{F}^{(1)}\left(\mathbf{G}_{1}\right)=\left(\frac{6}{15}, \frac{9}{15}\right)=\left(\frac{2}{5}, \frac{3}{5}\right), H_{F}^{(2)}\left(\mathbf{G}_{1}\right)=\left(\frac{26}{63}, \frac{37}{63}\right), H_{F}^{(3)}\left(\mathbf{G}_{1}\right)=$ $\left(\frac{106}{255}, \frac{149}{255}\right) \ldots$. As we show in Fig. $5, H_{F}^{(1)}\left(\mathbf{G}_{1}\right)$ corresponds to the second disk of the period-doubling cascade. All the other F-harmonics $H_{F}^{(2)}\left(\mathbf{G}_{1}\right)$, $H_{F}^{(3)}\left(\mathbf{G}_{1}\right) \ldots$ are cardioids placed in the chaotic band $\mathbf{B}_{1}$ and are the last appearance cardioids of this band. Hence, the F-harmonics of the gene $\mathbf{G}_{1}$ generate the structure of the period $-2^{1}$ chaotic band $\mathbf{B}_{1}$. The limit of this band is $H_{F}^{(\infty)}\left(\mathbf{G}_{1}\right)=\left(\frac{5}{12}, \frac{7}{12}\right)$ corresponding to the Misiurewicz point $m_{1}=\left(\frac{5}{12}, \frac{7}{12}\right)$ located at $c=-1.543689012 \ldots$ that separates the period- 1 chaotic bands $\mathbf{B}_{0}$ and $\mathbf{B}_{1}$ (see Fig. 6).

If we consider the new "bridge pattern", which is the second disk of the period-doubling cascade, as a gene $\mathbf{G}_{2}=\left(\frac{2}{5}, \frac{3}{5}\right)$ (see Fig. 5), we have that the F-harmonics of $\mathbf{G}_{2}$ generate the external arguments of the structure of the period- $2^{2}$ chaotic band $\mathbf{B}_{2}$ (and another bridge pattern that will be the third disk of the period-doubling cascade, the $\mathbf{G}_{3}=\left(\frac{7}{17}, \frac{10}{17}\right)$ ). The limit of the band $\mathbf{B}_{2}$ corresponds to the Misiurewicz point $m_{2}=\left(\frac{33}{80}, \frac{47}{80}\right)$, that separates the chaotic bands $\mathbf{B}_{1}$ and $\mathbf{B}_{2}$ (see Fig. 6). Let us see that in Fig. 5 we always depict the irreducible form of the arguments, although we have to use the normalized form to operate with Eq. (6). 
Note that there is a doubling operation mapping the chaotic band $\mathbf{B}_{0}$ onto the chaotic band $\mathbf{B}_{1}$. Here the binary expansion of an external angle is modified in such a way that every 0 is replaced by 01 and every 1 is replaced by 10 , according to $\mathbf{G}_{1}=\left(\frac{1}{3}, \frac{2}{3}\right)=(. \overline{01}, . \overline{10})$, for both hyperbolic components and Misiurewicz points. For example, $H_{F}^{(2)}\left(\mathbf{G}_{0}\right)=\left(\frac{3}{7}, \frac{4}{7}\right)=(. \overline{011}, \overline{100})$ and $H_{F}^{(2)}\left(\mathbf{G}_{1}\right)=\left(\frac{26}{63}, \frac{27}{63}\right)=(. \overline{011010}, . \overline{100101}) ; m_{0}=\left(\frac{1}{2}, \frac{1}{2}\right)=(.0 \overline{1}, .1 \overline{0})$ and $m_{1}=$ $\left(\frac{5}{12}, \frac{7}{12}\right)=(.01 \overline{10}, .10 \overline{01})$. In the same way there is a doubling operation mapping the chaotic band $\mathbf{B}_{0}$ onto $\mathbf{B}_{2}$. Here the binary expansion of an external angle is modified in such a way that every 0 is replaced by 0110 and every 1 is replaced by 1001 , according to $\mathbf{G}_{2}=\left(\frac{2}{5}, \frac{3}{5}\right)=(\overline{0110}, . \overline{1001})$, for both hyperbolic components and Misiurewicz points. For example, $H_{F}^{(2)}\left(\mathbf{G}_{0}\right)=\left(\frac{3}{7}, \frac{4}{7}\right)=$ $(\overline{011}, \overline{100})$ and $H_{F}^{(2)}\left(\mathbf{G}_{2}\right)=\left(\frac{563}{1365}, \frac{802}{1365}\right)=(. \overline{011010011001}, . \overline{100101100110})$; $m_{0}=\left(\frac{1}{2}, \frac{1}{2}\right)=(.0 \overline{1}, .1 \overline{0})$ and $m_{2}=\left(\frac{33}{80}, \frac{47}{80}\right)=(.0110 \overline{1001}, .1001 \overline{0110})$, and so on.

Generalizing, by applying Eq. (6), the F-harmonics of the disk $n$ of the perioddoubling cascade, the gene $\mathbf{G}_{n}$, generates the external arguments of the last appearance cardioids of the period- $2^{n}$ chaotic band $\mathbf{B}_{n}$, and a bridge disk of the period-doubling cascade, the gene $\mathbf{G}_{n+1}=H_{F}^{(1)}\left(\mathbf{G}_{n}\right)$. Likewise, $H_{F}^{(\infty)}\left(\mathbf{G}_{n}\right)$ is a Misiurewicz point $m_{n}$, a primary separator (or band-merging point) of the chaotic bands $\mathbf{B}_{n-1}$ and $\mathbf{B}_{n}$. The external arguments of $m_{n}$ can be calculated using Eq. (7).

This double process (disks and chaotic bands generation) continues indefinitely, and both meet at the Myrberg-Feigenbaum point, periodic disks on the right and chaotic bands on the left. Thanks to this procedure the whole harmonic structure of a 1D quadratic map was rigorously calculated in [23] by using symbolic sequences and now by using external arguments. In the same way as we have calculated the external arguments of the harmonic structure of the main cardioid, we can calculate the external arguments of the harmonic structure of anyone of the antenna midgets.

\section{Composition of external arguments}

By using the tools we have introduced up to now, we can calculate the external arguments of the structural components and the Misiurewicz points being tips or being merging points. That is what we denominate the structure of the Mandelbrot set antenna, that constitutes its framework or skeleton. But for each one of these structural components and Misiurewicz points there are an infinity more that have not been treated so far. In [23] we introduced other composition rules of simbolic sequences, and in [24] we introduced the heredity 
concept that make possible to calculate the descendants of any hyperbolic component if we know all the ancestors of such a hyperbolic component. Let us see that the above considerations can be applied to the calculation of external arguments in the Mandelbrot set antenna.

First of all, let us consider a midget of the Mandelbrot set antenna. Let $c$ be the parameter value of the midget center, and let $\left(\theta_{1}, \theta_{2}\right)$ be the pair of external arguments of the midget cusp point. The symbolic sequence (obtained from the orbit of $c$ ) and the kneading sequence [35, 36] (obtained from $\theta_{1}$ ) are equivalent. For example, let us consider the period-12 midget located at $c=-1.64127850 \ldots$ with external arguments $\left(\frac{1738}{4095}, \frac{2357}{4095}\right)$. The symbolic sequence of $c$ is CLRLLRLRLLLL and the kneading sequence of $\frac{1738}{4095}$ is $\overline{A B A A B A B A A A A *_{2}}$, the same if we do $\mathrm{L}=A, \mathrm{R}=B, \mathrm{C}=*_{2}$ and write the letter " $C$ " of the symbolic sequence at the end.

As is also known, there is an easy algorithm turning symbolic sequences (kneading sequences) into binary expansions and vice versa. There are two external arguments for each hyperbolic component. Since in the Mandelbrot set antenna the addition of these two external arguments equals to 1 , if we know the binary expansion of one of them we can obtain the binary expansion of the other by simply interchanging all the "0" and " 1 ". For our convenience, we always use the binary expansion whose first digit is a " 0 ". Let us see first the conversion from a symbolic sequence to a binary expansion. The first digit of the binary expansion, the corresponding to the " $\mathrm{C}$ ", is " 0 " and the following binary digits will be " 1 " or " 0 " if the L-parity of the part of the symbolic sequence until the digit (included) is odd or even respectively. Let us see now the conversion from a binary expansion, whose first digit is a "0", to a symbolic sequence. The first letter of the symbolic sequence is "C", and the following letters will be "L" or "R" in such a way that the L-parity of the part of the symbolic sequence until the letter (included) is odd when the binary digit is " 1 " or is even when the binary digit is " 0 ".

Taking into account the preceding paragraphs it is easy to see that the composition of two symbolic sequences (toward the left or toward the right) in [23] can be applied to the calculation of external arguments in the Mandelbrot set antenna. Let $\left(. \overline{a_{1}}, . \overline{a_{2}}\right)$ be the binary expansions of the external arguments of the augend, and let $\left(. \overline{a_{1}^{\prime}}, \overline{a_{2}^{\prime}}\right)$ be the binary expansions of the external arguments of the addend. The composition toward the left, $\left(. \overline{a_{1}}, . \overline{a_{2}}\right) \overleftarrow{+}\left(. \overline{a_{1}^{\prime}}, \overline{a_{2}^{\prime}}\right)$, and the composition toward the right, $\left(. \overline{a_{1}}, \overline{a_{2}}\right) \overrightarrow{+}\left(. \overline{a_{1}^{\prime}}, \overline{a_{2}^{\prime}}\right)$, can be formulated as follows:

$$
\left(. \overline{a_{1}}, \overline{a_{2}}\right) \overleftarrow{+}\left(. \overline{a_{1}^{\prime}}, \overline{a_{2}^{\prime}}\right)=\left(. \overline{a_{1} a_{2}^{\prime}}, \overline{a_{2} a_{1}^{\prime}}\right)
$$




$$
\left(. \overline{a_{1}}, \overline{a_{2}}\right) \overrightarrow{+}\left(. \overline{a_{1}^{\prime}}, . \overline{a_{2}^{\prime}}\right)=\left(\overline{a_{1} a_{1}^{\prime}}, \overline{a_{2} a_{2}^{\prime}}\right)
$$

Equations (8) can be also written in the normalized external arguments form as follows:

$$
\begin{aligned}
& \left(\frac{a_{1}}{2^{p}-1}, \frac{a_{2}}{2^{p}-1}\right) \overleftarrow{+}\left(\frac{a_{1}^{\prime}}{2^{p^{\prime}}-1}, \frac{a_{2}^{\prime}}{2^{p^{\prime}}-1}\right)=\left(\frac{2^{p^{\prime}} a_{1}+a_{2}^{\prime}}{2^{\left(p+p^{\prime}\right)}-1}, \frac{2^{p^{\prime}} a_{2}+a_{1}^{\prime}}{2^{\left(p+p^{\prime}\right)}-1}\right) \\
& \left(\frac{a_{1}}{2^{p}-1}, \frac{a_{2}}{2^{p}-1}\right) \rightarrow\left(\frac{a_{1}^{\prime}}{2^{p^{\prime}}-1}, \frac{a_{2}^{\prime}}{2^{p^{\prime}}-1}\right)=\left(\frac{2^{p^{\prime}} a_{1}+a_{1}^{\prime}}{2^{\left(p+p^{\prime}\right)}-1}, \frac{2^{p^{\prime}} a_{2}+a_{2}^{\prime}}{2^{\left(p+p^{\prime}\right)}-1}\right) .
\end{aligned}
$$

The composition of two legal external arguments (see Section 9) is not always possible. We use this composition to calculate the external arguments of the descendants of the augend (see section 9). We wonder if this composition is possible in any case. We do not know a necessary and sufficient condition that responds to this question. However, a sufficient condition is that the addend is a heredity transmitter of the augend [24], or a composition of the heredity transmitters of the augend.

\section{Example}

Let the augend be the pair of normalized external arguments $\left(\frac{13}{31}, \frac{18}{31}\right)$ that correspond to the period- 5 hyperbolic component $\alpha_{3}$ of the Fig. 7, and let the addend be the period-2 disk of the period doubling cascade whose external arguments are $\left(\frac{1}{3}, \frac{2}{3}\right)$. We have $\left(\frac{13}{31}, \frac{18}{31}\right) \overleftarrow{+}\left(\frac{1}{3}, \frac{2}{3}\right)=\left(\frac{54}{127}, \frac{73}{127}\right)$ that correspond to the normalized external arguments of the period- 7 hyperbolic component $7 a$ on the left of $\alpha_{3}$. Likewise, we have $\left(\frac{13}{31}, \frac{18}{31}\right) \overrightarrow{+}\left(\frac{1}{3}, \frac{2}{3}\right)=\left(\frac{53}{127}, \frac{74}{127}\right)$ that correspond to the normalized external arguments of the period-7 hyperbolic component $7 b$ on the right of $\alpha_{3}$.

\section{External arguments of a Misiurewicz point}

Let $\left(\frac{a_{1}}{2^{p}-1}, \frac{a_{2}}{2^{p}-1}\right)$ be the normalized external arguments of a hyperbolic component and let $\left(\frac{a_{1}^{\prime}}{2^{p^{\prime}}-1}, \frac{a_{2}^{\prime}}{2^{p^{\prime}}-1}\right)$ be the normalized external arguments of a heredity transmitter ancestor [24]. The external arguments of the leftward composition of these pairs of external arguments can be calculated according to Eq. (9). Likewise by applying Eq. (9), the external arguments of the leftward composition of the former result with the same heredity transmitter ancestor can also be calculated. If we repeat the process, in the limit, these infinite leftward 

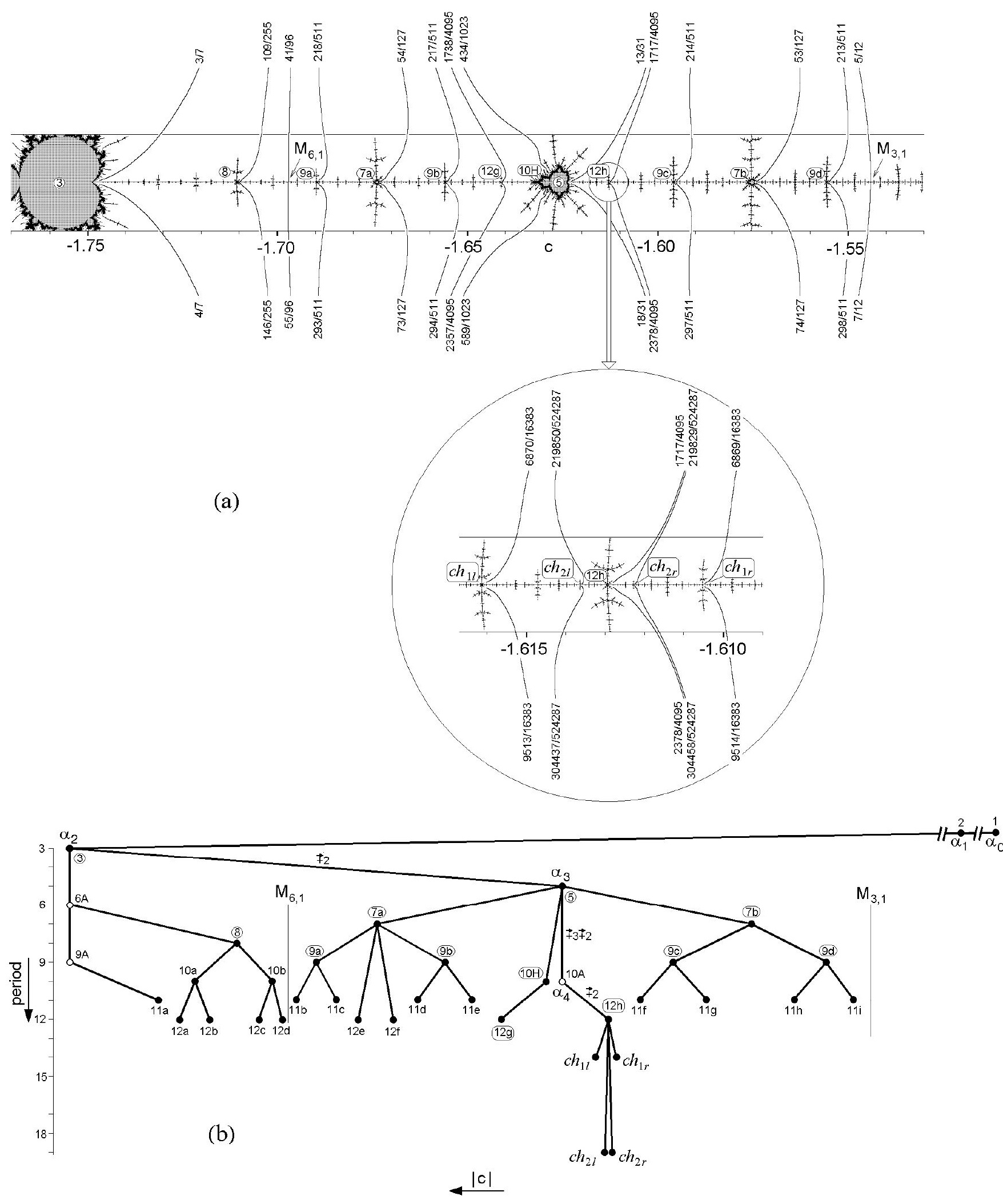

Fig. 7. (a) External arguments at the neighborhood of the period-5 hyperbolic component located on the right of the period-3 hyperbolic component in the Mandelbrot set antenna. (b) Nonbinary tree model of (a), showing the ancestors and children of the " $12 h$ " hyperbolic component. 
compositions give the external arguments of a Misiurewicz point located on the left of the hyperbolic component. If the heredity transmitter ancestor is the first harmonic of the gene of the chaotic band where the hyperbolic component is placed, the Misiurewicz point in a characteristic one (it has the same period as the chaotic band) [21]. Otherwise, is a non-characteristic one [21]. As it is easy to prove, the former infinite repetition of the leftward composition gives

$$
\begin{gathered}
{\left[\left(\frac{a_{1}}{2^{p}-1}, \frac{a_{2}}{2^{p}-1}\right) \overleftarrow{+}\left(\frac{a_{1}^{\prime}}{2^{p^{\prime}}-1}, \frac{a_{2}^{\prime}}{2^{p^{\prime}}-1}\right)\right] \overleftarrow{+}} \\
\overleftarrow{+}\left(\frac{a_{1}^{\prime}}{2^{p^{\prime}}-1}, \frac{a_{2}^{\prime}}{2^{p^{\prime}}-1}\right) \overleftarrow{+} \ldots=\left(\frac{a_{1}+\frac{a_{2}^{\prime}}{2^{p^{\prime}}-1}}{2^{p}}, \frac{a_{2}+\frac{a_{1}^{\prime}}{2^{p^{\prime}}-1}}{2^{p}}\right) .
\end{gathered}
$$

In the same way, infinite rightward compositions give the external arguments of a Misiurewicz point located on the right of the hyperbolic component. We have:

$$
\begin{gathered}
{\left[\left(\frac{a_{1}}{2^{p}-1}, \frac{a_{2}}{2^{p}-1}\right) \rightarrow\left(\frac{a_{1}^{\prime}}{2^{p^{\prime}}-1}, \frac{a_{2}^{\prime}}{2^{p^{\prime}}-1}\right)\right] \overrightarrow{+}} \\
\overrightarrow{+}\left(\frac{a_{1}^{\prime}}{2^{p^{\prime}}-1}, \frac{a_{2}^{\prime}}{2^{p^{\prime}}-1}\right) \overrightarrow{+} \ldots=\left(\frac{a_{1}+\frac{a_{1}^{\prime}}{2^{p^{\prime}}-1}}{2^{p}}, \frac{a_{2}+\frac{a_{2}^{\prime}}{2^{p^{\prime}}-1}}{2^{p}}\right) .
\end{gathered}
$$

\section{Example}

The limit of the rightward composition of the external arguments of the period3 hyperbolic component $\left(\frac{3}{7}, \frac{4}{7}\right)$ with the external arguments of its heredity transmitter ancestor $\left(\frac{1}{3}, \frac{2}{3}\right)$ is $\left[\left[\left(\frac{3}{7}, \frac{4}{7}\right) \overrightarrow{+}\left(\frac{1}{3}, \frac{2}{3}\right)\right] \overrightarrow{+}\left(\frac{1}{3}, \frac{2}{3}\right)\right] \overrightarrow{+} \ldots=\left(\frac{5}{12}, \frac{7}{12}\right)$, which in Fig. 7 is the Misiurewicz point $M_{3,1}$ with preperiod 3 and period 1 [26]. Note that we obtain the symbolic sequence of a point $c$ of the Mandelbrot set antenna from the orbit $0, c, c^{2}+c \ldots$ of the critical point of $z^{2}+c$. Other authors, for example [37], obtain the symbolic sequence from the orbit $c, c^{2}+c \ldots$ of the critical value of $z^{2}+c$. Thus, we write the symbolic sequence of the period-3 midget of the Mandelbrot set antenna as CLR, whereas other authors write LRC. In both cases the number of letters of the symbolic sequence coincides with the period of the orbit, and the only difference is the position of the letter "C". However, the result is different when the parameter value of a Misiurewicz point is iterated. Then, the periods obtained in both cases are the same whereas the preperiods differ in one unity.

\section{Example}

The limit of the leftward composition of the external arguments of the period5 hyperbolic component $\left(\frac{13}{31}, \frac{18}{31}\right)$ with the external arguments of its heredity 
transmitter ancestor $\left(\frac{1}{3}, \frac{2}{3}\right)$ is $\left[\left[\left(\frac{13}{31}, \frac{18}{31}\right) \overleftarrow{+}\left(\frac{1}{3}, \frac{2}{3}\right)\right] \overleftarrow{+}\left(\frac{1}{3}, \frac{2}{3}\right)\right] \overleftarrow{+} \ldots=\left(\frac{41}{96}, \frac{55}{96}\right)$ which in Fig. 7 is the Misiurewicz point $M_{6,1}[26]$.

\section{Autocomposition}

Let us consider the case of the leftward autocomposition of the external arguments of a period- $p$ hyperbolic component. In such a manner we obtain the external arguments of the first F-harmonic of the hyperbolic component. Indeed:

$$
\left(\frac{a_{1}}{2^{p}-1}, \frac{a_{2}}{2^{p}-1}\right) \overleftarrow{+}\left(\frac{a_{1}}{2^{p}-1}, \frac{a_{2}}{2^{p}-1}\right)=\left(\frac{a_{1} 2^{p}+a_{2}}{2^{2 p}-1}, \frac{a_{2} 2^{p}+a_{1}}{2^{2 p}-1}\right)
$$

but also

$$
H_{F}^{(1)}\left(\frac{a_{1}}{2^{p}-1}, \frac{a_{2}}{2^{p}-1}\right)=\left(\frac{a_{1} 2^{p}+a_{2}}{2^{2 p}-1}, \frac{a_{2} 2^{p}+a_{1}}{2^{2 p}-1}\right)
$$

If we apply this autocomposition again and again we obtain the successive F-harmonics. I.e., the order $i$ F-harmonic of a pair of normalized external arguments is also the order $i$ autocomposition of the same pair.

Let us consider now the case of the rightward autocomposition of the external arguments of a period- $p$ hyperbolic component. As we can see, with this autocomposition we obtain first a period- $2 p$ hyperbolic component, the first antiharmonic of the period- $p$ hyperbolic component, but, after an elemental simplification, we obtain again the period- $p$ hyperbolic component. Indeed

$$
\begin{gathered}
\left(\frac{a_{1}}{2^{p}-1}, \frac{a_{2}}{2^{p}-1}\right)+\left(\frac{a_{1}}{2^{p}-1}, \frac{a_{2}}{2^{p}-1}\right)= \\
=\left(\frac{a_{1}\left(2^{p}+1\right)}{2^{2 p}-1}, \frac{a_{2}\left(2^{p}+1\right)}{2^{2 p}-1}\right)=\left(\frac{a_{1}}{2^{p}-1}, \frac{a_{2}}{2^{p}-1}\right) .
\end{gathered}
$$

Antiharmonics have already been introduced by Metropolis Stein and Stein in 1973 [31]. Normally, it is thought that antiharmonics have no real existence. However, we reckon that this idea must be revised because, although we have the same value when we simplify, if we do not simplify we have a body, the first antiharmonic, with a period which is double than the original one. Therefore, even though placed on the same location, it is different. Besides, it is important to study the antiharmonics because, as we saw in [24], they can be heredity transmitters. 


\section{Example}

Let us calculate the first harmonic and the first antiharmonic, see Fig. 7, of the period-5 hyperbolic component whose external arguments are $\left(\frac{13}{31}, \frac{18}{31}\right)$. In order to do so, we apply Eqs. (12) and (13) obtaining $\left(\frac{13}{31}, \frac{18}{31}\right) \overleftarrow{\leftarrow}\left(\frac{13}{31}, \frac{18}{31}\right)=$ $\left(\frac{434}{1023}, \frac{589}{1023}\right)$, the harmonic $10 \mathrm{H}$ in Fig. 7 , and $\left(\frac{13}{31}, \frac{18}{31}\right) \overrightarrow{+}\left(\frac{13}{31}, \frac{18}{31}\right)=$ $\left(\frac{429}{1023}, \frac{594}{1023}\right)=\left(\frac{13}{31}, \frac{18}{31}\right)$, the antiharmonic 10A in Fig. $7(\mathrm{~b})$, given in both normalized and irreducible forms. As expected, its normalized form does not correspond to any period-10 existing antenna hyperbolic component. However, its irreducible form coincides with the normalized form of the period- 5 original hyperbolic component.

\section{Ancestral decomposition of external arguments}

As we have seen in [24], the pattern of a hyperbolic component of a 1D quadratic map can be expressed as the addition of the gene of the chaotic band where the pattern is, and the heredity transmitters related to the ancestral path that joins all its ancestors. We can see that if we manage external arguments of the Mandelbrot set antenna hyperbolic components, the same happens.

The normalized external arguments of a period- $p$ hyperbolic component of the Mandelbrot set have the form $\left(\frac{a_{1}}{2^{p}-1}, \frac{a_{2}}{2^{p}-1}\right)$. However, an antenna hyperbolic component has not arbitrary values $a_{1}$ and $a_{2}$. The possible values are very scarce if we impose the condition $\frac{a_{1}}{2^{p}-1}+\frac{a_{2}}{2^{p}-1}=1$, which points out that the hyperbolic component is on the antenna. Nevertheless, still there are some values of $a_{1}$ and $a_{2}$ that, even if they verify this last condition, do not correspond to any antenna hyperbolic component. When this happens, we say that the pair of external arguments are non-legal external arguments. Therefore, we can say that legal external arguments are a pair of external arguments where $a_{1}$ and $a_{2}$ correspond to existing values of a period- $p$ antenna hyperbolic component.

We can study the Mandelbrot set antenna as an extension of the quadratic map $x_{n+1}=x_{n}^{2}+c$ and this we have called real Mandelbrot map. As we saw in $[23,24]$, starting from the symbolic sequence $\mathrm{C}$ of the superstable period1 orbit of the real Mandelbrot map and applying the composition rules and the heredity concept, it is possible to obtain all and each of the symbolic sequences of the superstable orbits. This result is based on a lot of computer experiments. The inverse process is also possible. Given a symbolic sequence, and without any need for other data, we can calculate the family tree of such a symbolic sequence. To this end, we carry out the ancestral decomposition of the symbolic sequence, obtaining its ancestors and heredity transmitters. 
We extend these experimental results to the Mandelbrot set antenna in the sense that the pair of legal external arguments of a hyperbolic component can always be decomposed in additions (toward the left or toward the right) of external arguments of structural components of the chaotic band where the hyperbolic component is placed. Next, by analyzing this decomposition, we can easily see the ancestral path and the heredity transmitters of the legal external arguments. We shall use as legality test the following conditions: the decomposition has to be possible, and the decomposition has to correspond to a valid ancestral path. When these conditions are verified, the external arguments are legal ones. If, on the contrary, the decomposition is not possible, or if the decomposition being possible it does not correspond to a valid ancestral path, then the external arguments are non-legal ones. Let us think in the inverse process: let us suppose that we know the external arguments of the $i$ structural components obtained from the decomposition of a pair of legal external arguments. Obviously, if we now compose them (by using the additions in the proper direction obtained from the decomposition) we obtain again the pair of legal external arguments of the beginning in the form

$$
\begin{gathered}
{\left[\cdots\left[\left(\frac{a_{1(1)}}{2^{p_{1}}-1}, \frac{a_{2(1)}}{2^{p_{1}}-1}\right) \stackrel{\leftrightarrow}{+}\left(\frac{a_{1(2)}}{2^{p_{2}}-1}, \frac{a_{2(2)}}{2^{p_{2}}-1}\right)\right] \stackrel{\leftrightarrow}{+} \cdots\right] \stackrel{\leftrightarrow}{+}} \\
\quad \stackrel{\leftrightarrow}{+}\left(\frac{a_{1(i)}}{2^{p_{i}}-1}, \frac{a_{2(i)}}{2^{p_{i}}-1}\right)=\left(\frac{a_{1}}{2^{p}-1}, \frac{a_{2}}{2^{p}-1}\right),
\end{gathered}
$$

where $p=p_{1}+p_{2}+\cdots+p_{i}$ and $\overleftrightarrow{+}$ is either $\overleftarrow{+}$ or $\overrightarrow{+}$. Let us see again the decomposition of a pair of external arguments. This decomposition has to verify Eq. (14). If what we know is the result, we have to test all the possible cases, discard all that do not lead to the solution, and take the only one that lead us to the correct result, i.e., we test all the possible $p=p_{1}+p_{2}+\cdots+p_{i}$. For each one of these decompositions we calculate the possible values of the Eq. (14) with all the additions towards the left and towards the right. This is very long and tedious if we do it manually, but it is elementary if we make it by using a computer program.

Once this decomposition is obtained, by taking into account the heredity rules that we saw in [24], it is easy to find the ancestral path that joins all the ancestors and determine the heredity transmitters. Now, if we compose the legal external arguments with their heredity transmitters we obtain all the "children" or first generation descendants. By applying the same procedure to each one of its children we obtain all the grandchildren. If we continue uninterruptedly this procedure we obtain the family tree.

\section{Example}

Let us calculate, see Fig. 7, the external arguments of the children of the period-12 hyperbolic component " $12 h$ " whose external arguments are 
$\left(\frac{1717}{2^{12}-1}, \frac{2378}{2^{12}-1}\right)=\left(\frac{1717}{4095}, \frac{2378}{4095}\right)$, which is a simplified case of the example of the section IV of [24]. Figure 7(a) shows a zone of the antenna with " $12 h$ " and its ancestors and descendants. Figure 7(b) shows a family tree model of Fig. $7(\mathrm{a})$.

Starting from the external arguments of " $12 h$ ", we calculate the decomposition of " $12 h$ " in structural components as we explained above. The obtained result is: $12=3 \overrightarrow{+} 2 \overrightarrow{+} 3 \overrightarrow{+} 2 \overrightarrow{+} 2$, where, instead of the structural components external arguments, we only indicate the periods and the addition directions. Let us see, with the help of Fig. 7(b) that with this result we can find the ancestral path, the ancestors and the heredity transmitters. When the heredity transmitters are known, we can calculate the descendants of " $12 h$ ".

The decomposition of " $12 h$ " starts from the period-3 structural component $\alpha_{2}=\left(\frac{3}{7}, \frac{4}{7}\right)$, of the chaotic band $\mathbf{B}_{0}$, though this is not the first component of the ancestral path. Before $\alpha_{2}$ we have the main cardioid $\alpha_{0}=\left(\frac{0}{1}, \frac{1}{1}\right)$ and the first disk of the period doubling cascade $\alpha_{1}=\left(\frac{1}{3}, \frac{2}{3}\right)$, that are not in $\mathbf{B}_{0}$, and therefore are not in the decomposition. To the period-3 hyperbolic component $\alpha_{2}$ we add rightwards its period- 2 ancestor $\alpha_{1}$ and we obtain the period-5 hyperbolic component of the ancestral path of " $12 h$ ", $\alpha_{3}=\left(\frac{3}{7}, \frac{4}{7}\right) \overrightarrow{+}\left(\frac{1}{3}, \frac{2}{3}\right)=$ $\left(\frac{13}{31}, \frac{18}{31}\right)$, as can be seen in Fig. 7 .

If we analyze the decomposition of " $12 h$ ", it seems that now we should compose rightwards this period- 5 hyperbolic component and the period-3 structural component. However, as we know from [24], even though the period3 hyperbolic component $\alpha_{2}$ is an ancestor of the period-5 hyperbolic component $\alpha_{3}, \alpha_{2}$ is not a heredity transmitter, and therefore this composition is not valid. But we can compose rightwards this period-5 hyperbolic component and $(\overrightarrow{+} 3 \overrightarrow{+} 2)$, i. e., we can compose rightwards this period-5 hyperbolic component and itself to give the antiharmonic. Hence we have: $\alpha_{4}=\left(\frac{13}{31}, \frac{18}{31}\right) \overrightarrow{+}\left(\frac{13}{31}, \frac{18}{31}\right)=\left(\frac{429}{1023}, \frac{594}{1023}\right)=\left(\frac{13}{31}, \frac{18}{31}\right)$, that is named $10 \mathrm{~A}$ in Fig. 7. Let us note that, since it is an antiharmonic, it has two normalized forms: the first one (period-10) really corresponds to the antiharmonic, and the second one (period-5) is the starting hyperbolic component.

As we can see in the decomposition of " $12 h$ ", we end next with the composition $\overrightarrow{+} 2$, that is a valid one, and we obtain the starting period-12 hyperbolic component $\left(\frac{429}{1023}, \frac{594}{1023}\right) \overrightarrow{+}\left(\frac{1}{3}, \frac{2}{3}\right)=\left(\frac{1717}{4095}, \frac{2378}{4095}\right)$ named "12h" in Fig. $7(\mathrm{~b})$.

Now, we want to calculate the first generation descendants (children) of " $12 h$ ". In order to do so, as we know from [24], we have to compose, both rightwards and leftwards, " $12 h$ " and all the heredity transmitters. Hence, we have to determine the heredity transmitters. The ancestral path of " $12 h$ " is $\alpha_{1}, \alpha_{2}, \alpha_{3}$ and $\alpha_{4}$, as we can see in Fig. $7(\mathrm{~b})$. The period-2 ancestor $\alpha_{1}=\left(\frac{1}{3}, \frac{2}{3}\right)$ is a heredity 
transmitter. The period-3 ancestor $\alpha_{2}=\left(\frac{3}{7}, \frac{4}{7}\right)$ is not a heredity transmitter. The period-5 ancestor $\alpha_{3}=\left(\frac{13}{31}, \frac{18}{31}\right)$ is neither a heredity transmitter, but its period-7 child " $7 b$ " with external arguments $\left(\frac{13}{31}, \frac{18}{31}\right) \overrightarrow{+}\left(\frac{1}{3}, \frac{2}{3}\right)=\left(\frac{53}{127}, \frac{74}{127}\right)$ is a heredity transmitter. The period-10 ancestor $\alpha_{4}=\left(\frac{429}{1023}, \frac{594}{1023}\right)$ is neither a heredity transmitter. The heredity transmitters of $\left(\frac{1717}{4095}, \frac{2378}{4095}\right)$ are $\left(\frac{1}{3}, \frac{2}{3}\right)$ and $\left(\frac{53}{127}, \frac{74}{127}\right)$.

We compose rightwards and leftwards $\left(\frac{1717}{4095}, \frac{2378}{4095}\right)$ and these two heredity transmitters in order to find the external arguments of the children of " $12 h$ ". Thus, we obtain:

$$
\begin{aligned}
& c h_{1 r}=\left(\frac{1717}{4095}, \frac{2378}{4095}\right)+\left(\frac{1}{3}, \frac{2}{3}\right)=\left(\frac{6869}{16383}, \frac{9514}{16383}\right), \\
& c h_{1 l}=\left(\frac{1717}{4095}, \frac{2378}{4095}\right) \overleftarrow{+}\left(\frac{1}{3}, \frac{2}{3}\right)=\left(\frac{6870}{16383}, \frac{9513}{16383}\right), \\
& c h_{2 r}=\left(\frac{1717}{4095}, \frac{2378}{4095}\right) \nrightarrow\left(\frac{53}{127}, \frac{74}{127}\right)=\left(\frac{219829}{524287}, \frac{304458}{524287}\right), \\
& \operatorname{ch}_{2 l}=\left(\frac{1717}{4095}, \frac{2378}{4095}\right) \overleftarrow{+}\left(\frac{53}{127}, \frac{74}{127}\right)=\left(\frac{219850}{524287}, \frac{304437}{524287}\right) .
\end{aligned}
$$

If we repeat now the proccess for each one of the children, we obtain the grandchildren, great-granchildren, and so on up to the infinity to obtain all the descendants or the family tree of $\mathrm{P}$.

\section{Conclusions}

In this paper we have introduced several tools in order to operate with external arguments in the Mandelbrot set antenna.

We have introduced the MSS-harmonics of the external arguments of a period$p$ hyperbolic component. The order $i$ MSS-harmonic calculates the external arguments of the $i^{\text {th }}$ disk of the period doubling cascade, whose period is $2^{i} p$, of such a period- $p$ hyperbolic component.

We have introduced the Fourier harmonics (or F-harmonics) of the external arguments of a period- $p$ hyperbolic component. The order $i$ F-harmonic calculates the external arguments of the last appearance cardioid in the corresponding chaotic band (except for the first harmonic that calculates the first disk of the period doubling cascade). The order $i$ F-harmonic of a period- $p$ hyperbolic component has a period $(i+1) p$.

Besides, we have studied the order $\infty$ harmonics of both, MSS-harmonics 
and F-harmonics. The order $\infty$ MSS-harmonic of the external arguments of a period- $p$ hyperbolic component is a Myrberg-Feigenbaum point, whose external arguments are irrational, that we do not consider here. The order $\infty$ F-harmonic of the external arguments of a period- $p$ hyperbolic component is a Misiurewicz point. The order $\infty$ F-harmonic of a cardioid is a tip, and the order $\infty$ F-harmonic of a disk is a merging-point.

We have introduced the leftward composition and the rightward composition of two external arguments. With these composition rules for external arguments, and by taking into account the concept of heredity, we can calculate all the external arguments that constitute the family tree of a pair of given external arguments.

\section{Acknowledgements}

This work was supported by Ministerio de Ciencia y Tecnología of Spain, research grant TIC2001-0586.

\section{References}

[1] B. B. Mandelbrot, in Nonlinear Dynamics, edited by R.H.G. Helleman, Annals of New York Acad. Sciences 357 (1980) 249.

[2] B.B. Mandelbrot, Physica D 7 (1983) 224.

[3] A. Douady and J.H. Hubbard, CR. Acad. Sci. Paris 294 (1982) 123.

[4] A. Douady and J.H. Hubbard, Étude Dynamique des Polynômes Complexes, Publ. Math. d'Orsay, Part I No. 84-02, 1984; Part II No. 85-04, 1985.

[5] A. Douady, in Chaotic Dynamics and Fractals, M. Barnsley and S. G. Demko eds., Academic Press, 1986, p. 155.

[6] A. Douady. invited contribution in H.-O. Peitgen and P.H. Richter, The Beauty of Fractals, Springer-Verlag, 1986, p. 161.

[7] B. Branner, in Chaos and Fractals, R. L. Devaney and L. Keen eds., Proc. Symp. Appl. Math. 39, AMS 1989, p. 75.

[8] H.-O. Peitgen and P.H. Richter, The Beauty of Fractals, Springer-Verlag, 1986, p. 63.

[9] H.-O. Peitgen, H. Jürgens and D. Saupe, Chaos and Fractals, SpringerVerlag, New York, 1992, p. 802.

[10] L. Carleson and T.W. Gamelin, Complex Dynamics, Springer, New York, 1993, p. 139.

[11] W. Jung, preprint in preparation (2001).

[12] W. Jung, mandel.exe, a DOS program available from: 
http://www.iram.rwth-aachen.de/ jung/, or http://www.math.sunysb.edu/.

[13] E. Lau, D. Schleicher, IMS Preprint 94-19 (1994).

[14] A. Douady, X. Buff, R.L. Devaney and P. Sentenac, in The Mandelbrot set, T. Lei ed., Cambridge University Press, 2000, p. 19.

[15] E. N. Lorenz, J. Atmos. Sci. 20 (1963) 130.

[16] D. Ruelle and F. Takens, Commun. Math. Phys. 20 (1971) 167.

[17] J. P. Crutchfield and K. Kaneco, in Directions in chaos Vol. II, B-L. Hao ed., World-Scientific, Singapore, 1987.

[18] K. R. Sreenivasan, in Frontiers in Fluid Mechanics, S. H. Davis and J. L. Lumley eds., Springer-Verlag, Berlin, 1985, p. 41.

[19] C. Beck, Physica D 125 (1999) 171.

[20] M. Misiurewicz and Z. Nitecki, Mem. Am. Math. Soc. 94, No. 456 (1991).

[21] M. Romera, G. Pastor and F. Montoya, Physica A 232 (1996) 517.

[22] G. Pastor, M. Romera and F. Montoya, Chaos, Solitons and Fractals 7 (1996) 565.

[23] G. Pastor, M. Romera and F. Montoya, Physical Review E 56 (1997) 1476.

[24] M. Romera, G. Pastor, G. Álvarez and F. Montoya, Physical Review E 58 (1998) 7214.

[25] G. Pastor, M. Romera, J. C. Sanz-Martín and F. Montoya, Physica A 256 (1998) 369.

[26] G. Pastor, M. Romera and F. Montoya, Physica A 232 (1996) 536.

[27] G. Pastor, M. Romera, G. Álvarez and F. Montoya, Physica A 292 (2001) 207.

[28] B.-L. Hao and W.-M. Zheng, Int. J. Mod. Phys. B 3 (1989) 235.

[29] W.-M. Zheng and B.-L. Hao, in Experimental Study and Characterization of Chaos, B-L. Hao ed., World-Scientific, Singapore, 1990, p. 364.

[30] J. Milnor and W. Thurston, in Dynamical systems, J. C. Alexander ed., Springer-Verlag, Berlin, Heidelberg, 1988.

[31] N. Metropolis, M. L. Stein and P. R. Stein , J. Comb. Theory 15 (1973) 25.

[32] R.L. Devaney, in Complex Dynamical Systems, R.L. Devaney ed., American Mathematical Society 1994, p. 23.

[33] P. J. Myrberg, Ann. Acad. Sci. Fenn-M 336/3 (1963) 1.

[34] M. J. Feigenbaum, J. Stat. Phys. 19 (1978) 25.

[35] D. Schleicher, IMS Preprint 1997/13 (1997).

[36] J. Hubbard and D. Schleicher, in Complex Dynamical Systems, R.L. Devaney ed., American Mathematical Society 1994, p. 155.

[37] B.-L. Hao and W.-M Zheng, Applied Symbolic Dynamics and Chaos, World-Scientific, Singapore, 1998, p. 23. 